\title{
A Case of Liponecrotic Pseudocyst after Breast Augmentation by Autologous Fat Injection
}

\section{Dae Hyun Kim, Kyung Sik Kim}

Department of Plastic and Reconstructive Surgery, Myong-Ji Hospital, Goyang, Korea
No potential conflict of interest relevant to this article was reported.

\begin{abstract}
A 21-year-old woman underwent bilateral augmentation mammoplasty by injection of an unknown volume of fat obtained via trochanteric and abdominal liposuction. The procedure was performed by a surgeon untrained in plastic surgery, at a clinic not affiliated with a hospital. Six months later, she presented to our clinic with a palpable left breast lump. Physical examination revealed a large firm mass occupying the entire upper outer quadrant of the left breast and a normal right breast. Breast ultrasound showed a large, well-defined isoechoic mass in the left upper outer breast. Considering her age, the patient underwent core needle biopsy, since the mass mimicked a phyllodes tumor on ultrasonography. The 14-gauge core needle biopsy demonstrated multiple lipid droplets with some white-yellowish tissue, caused by liquefaction of the injected fat. Histopathologic examination demonstrated the presence of a pseudocyst with fat necrosis, granulomatous reaction to lipid material, and cystic formations containing oily fluid. No signs of malignancy were detected. Surgical excision was performed, and histopathology revealed findings consistent with fat necrosis.
\end{abstract}

Keywords Complication, Fat necrosis, Mammoplasty

\section{INTRODUCTION}

Autologous fat transplantation procedures for breast augmentation have become increasing utilized because of aesthetic demands [1]. Fat transplantation may be considered as an alternative to implants because it avoids complications related to implant insertion, such as hemorrhage, infection, and implant rupture or contracture, and because it does not inhibit breast parenchymal visibility on diagnostic modalities, including mammography or ultrasound [1,2]. However, autologous fat transplantation is associated with such drawbacks as a limited increase in size that can be achieved, the possibility of fat necrosis (producing oil cysts, calcification, and/or scarring), and relatively unpredictable outcomes $[1,3]$.

Received: Jul 29, 2015 Revised: Aug 31, 2015 Accepted: Sep 3, 2015 Correspondence: Kyung Sik Kim Department of Plastic \& Reconstructive Surgery, Myong-Ji Hospital, 55 Hwasu-ro 14beon-gil, Deokyang-gu, Goyang 10475, Korea. E-mail: kskimps@mjh.or.kr

Copyright $\odot 2015$ The Korean Society for Aesthetic Plastic Surgery.

This is an Open Access article distributed under the terms of the Creative Commons Attribution Non-Commercial License (http://creativecommons.org/licenses/by-nc/3.0/) which permits unrestricted non-commercial use, distribution, and reproduction in any medium, provided the original work is properly cited. www.e-aaps.org
In 1987, the American Society of Plastic and Reconstructive Surgeons [4] indicated that complications associated with autologous fat transplantation for breast augmentation might interfere with breast cancer surveillance/screening and should be prohibited. Yet, autologous fat grafting to the breast remains a widely-used procedure [1]. Furthermore, it is often performed by untrained individuals, which increases the likelihood of complications [1].

Here we present our experience with a liponecrotic pseudocyst that developed in the breast of a patient who underwent autologous fat transplantation by a surgeon who was not trained in plastic surgery.

\section{CASE REPORT}

A 21-year-old woman underwent bilateral augmentation mammoplasty by injection of an unknown volume of fat after trochanteric and abdominal liposuction. The procedure was performed by a surgeon not trained in plastic surgery, at a clinic not affiliated with a hospital. Six months later, the patient presented to our clinic with a palpable lump in her left breast. Physical examination revealed a large firm mass occupying all of the upper outer quadrant of the left breast and a normal right breast (Fig. 1). Breast ultrasound show- 


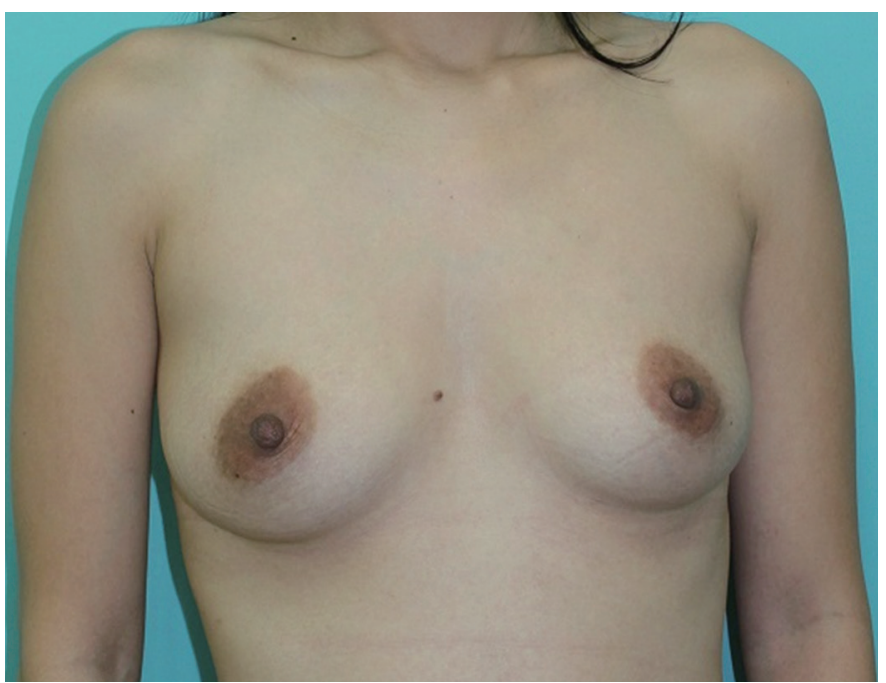

Fig. 1. Preoperative photograph showing a palpable mass in the left breast. A mild bulging contour is noted.

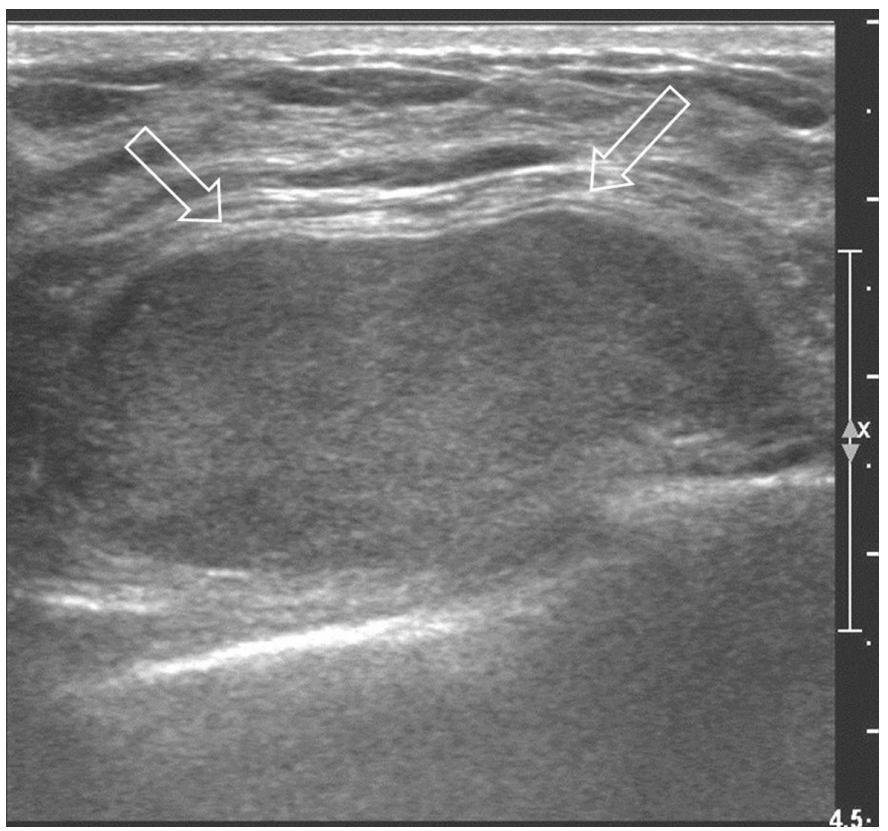

Fig. 2. Ultrasound of the left breast showing a large well-defined isoechoic lesion (arrow).

ed a large, well-defined isoechoic mass lesion in the left upper outer breast. Multiple variable-sized solid and cystic masses were also evident along both the internal and lateral mammary lymphatic chains (Fig. 2). Contrast-enhanced chest computed tomography revealed a large fatty mass embedded deep to the left pectoralis major muscle, and multiple subcutaneous nodules along both the lateral and internal mammary lymphatic chains (Fig. 3). Considering her age, the patient underwent core needle biopsy, since the mass mimicked a phyllodes tumor on ultrasonography. The 14-gauge core needle biopsy demonstrated multiple lipid droplets with some

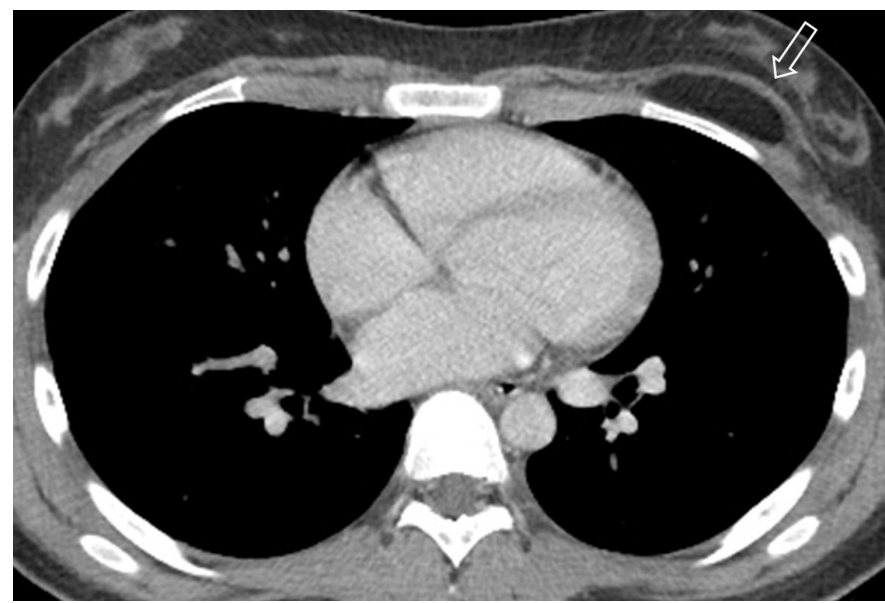

Fig. 3. Chest computed tomography scan of the left breast showing a fatty mass under the left pectoralis muscle (arrow).

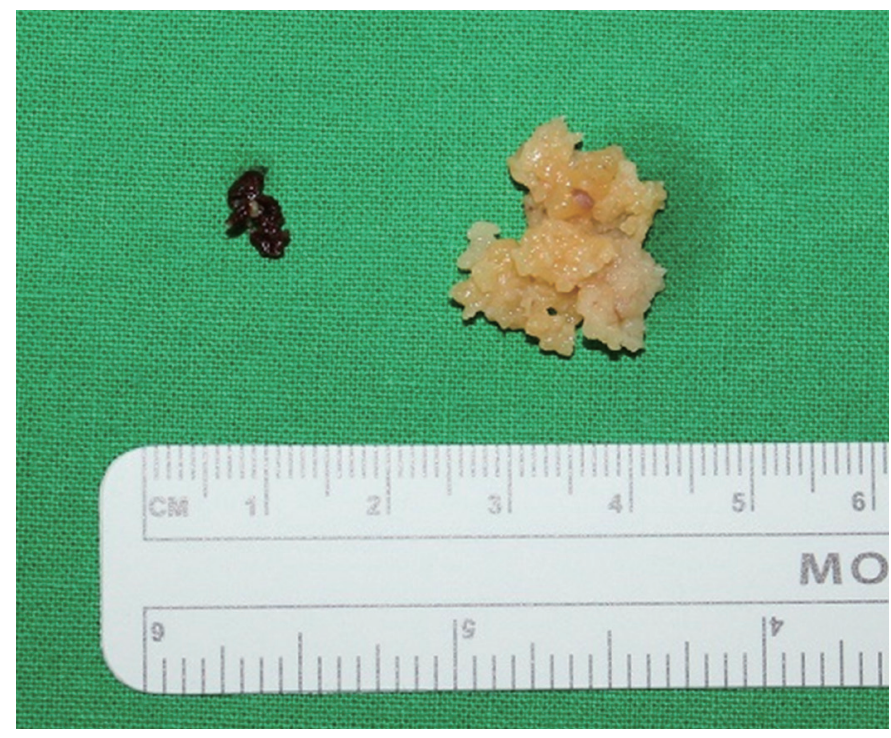

Fig. 4. Intraoperative view of extirpated specimens.

white-yellowish tissue, caused by liquefaction of the injected fat. Histopathologic examination demonstrated a pseudocyst with fibrotic walls (not shown), representing an area of fat necrosis containing oily fluid. No signs of malignancy were detected. Surgical excision was performed, and the histopathology revealed devitalized adipocytes, consistent with fat necrosis (Fig. 4 and 5).

\section{DISCUSSION}

Autologous fat transplantation is a widely-performed procedure, intended to improve facial and body contour depressions and scars and to augment lip, penis, and breast tissue [3]. Variable results have been reported, depending on the fat harvesting technique, injected volume, and injected area $[1-3,5]$. The major reported complica- 


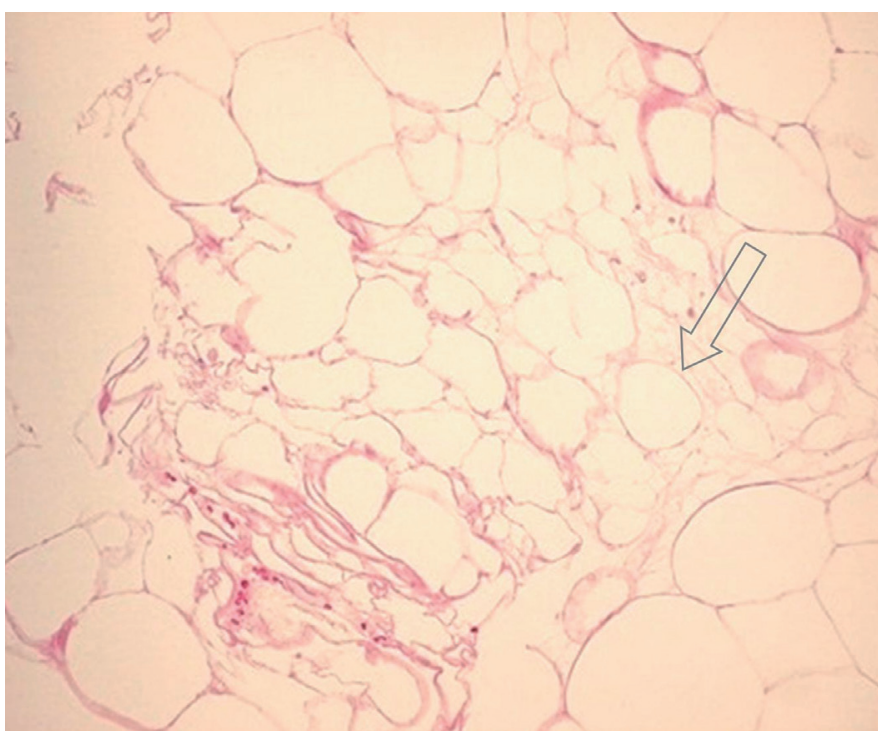

Fig. 5. Histopathologic examination (Hematoxylin and Eosin [H\&E], $\times 40$ ) showing shadowy outlines of dead adipocytes, indicating fat necrosis (arrow), without demonstrable endothelium lining the pseudocyst.

tions are hematomas or edema, infection, abscesses, chronic inflammation including granulomas, and resorption of the injected fat $[1,3]$.

Use of autologous fat transplantation in some locations, including the breast, remains contentious [4,6,7]. Recent technical improvements in autologous fat transplantation have increased confidence in the outcomes, and the procedure is now considered to be a potential alternative to artificial breast implants $[1,6,8]$. However, limitations that continue to be associated with autologous fat transplantation include the limited size increase, potential for fat necrosis (with oil cysts, calcification, and/or scarring), and unpredictable outcomes of the transferred material. The latter drawback can result in low graft survival and/or a longer procedure time [1-3,5].

The development of liponecrotic pseudocysts after fat injection has been previously described $[9,10]$. Injection of autologous nonvascularized fat lobules into breast tissue can cause fat necrosis and provoke an inflammatory reaction [1-3,5,7,10]. Generally, pseudocysts appear as cystic lesions on imaging studies, but they lack epithelium or endothelium histopathologically. Liponecrotic pseudocysts contain oily fluid or chalky white areas resulting from the combination of newly-formed free fatty acids with calcium in the cystic lesion. Similar to conventional pseudocysts, liponecrotic pseudocysts also lack epithelium or endothelium.

The injection of small volumes of fat may produce irregularlyshaped microcalcifications indistinguishable from carcinoma on mammography $[1,2,5,8,10]$. Injection of large volumes may cause the formation of multiple oil cysts and nodules (granulomas), which can interfere with breast examination and mimic neoplastic lesions $[1,2,4,5,7-10]$. Diagnostic needle biopsy is controversial and should be performed cautiously because of the risk of oil leakage, which can produce further inflammation and granuloma formation [3]. Excisional biopsy may be troublesome because of the adherent nature of pseudocysts and their lack of a true capsule [1-3,7,10]. Excision of adjacent breast tissue may occur, creating a breast deformity $[1-3,7,8,10]$.

Autologous fat transplantation to the breast requires close clinical and radiologic follow-up [1,8]. Any suspicious mass or abnormal radiologic finding must be histopathologically evaluated $[1,8]$. Long-term follow-up of breast autologous fat transplantation remains poorly studied. Such studies will be needed before the technique is introduced into routine practice.

In conclusion, this report describes a patient who developed a large liponecrotic pseudocyst in the breast 6 months after fat injection following trochanteric and abdominal liposuction. The possibility of pseudocysts after breast autologous fat injection is a complication that requires caution when using this technique for breast augmentation.

\section{PATIENT CONSENT}

Patients provided written consent for the use of their images.

\section{REFERENCES}

1. Hyakusoku H, Ogawa R, Ono S, et al. Complications after autologous fat injection to the breast. Plast Reconstr Surg 2009;123:360-70.

2. Coleman SR, Saboeiro AP. Fat grafting to the breast revisited: safety and efficacy. Plast Reconstr Surg 2007;119:775-85.

3. Har-Shai Y, Lindenbaum E, Ben-Itzhak O, et al. Large liponecrotic pseudocyst formation following cheek augmentation by fat injection. Aesthetic Plast Surg 1996;20:417-9.

4. ASPRS Ad-Hoc Committee. Report on autologous fat transplantation. ASPRS Ad-Hoc Committee on New Procedures, September 30, 1987. Plast Surg Nurs 1987;7:140-1.

5. Spear SL, Wilson HB, Lockwood MD. Fat injection to correct contour deformities in the reconstructed breast. Plast Reconstr Surg 2005;116: 1300-5.

6. Bircoll M. Cosmetic breast augmentation utilizing autologous fat and liposuction techniques. Plast Reconstr Surg 1987;79:267-71.

7. Hartrampf CR Jr, Bennett GK. Autologous fat from liposuction for breast augmentation. Plast Reconstr Surg 1987;80:646.

8. Veber M, Tourasse C, Toussoun G, et al. Radiographic findings after breast augmentation by autologous fat transfer. Plast Reconstr Surg 2011;127:1289-99.

9. Maillard GF. Liponecrotic cysts after augmentation mammaplasty with fat injections. Aesthetic Plast Surg 1994;18:405-6.

10. Montañana Vizcaino J, Baena Montilla P, Benito Ruiz J. Complications of autografting fat obtained by liposuction. Plast Reconstr Surg 1990; 85:638-9. 